\title{
Differential expression of Wnt13 isoforms during leukemic cell differentiation
}

\author{
RODICA PETRUTA BUNACIU ${ }^{1}$, TAO TANG and CATHERINE D. MAO \\ Graduate Center for Nutritional Sciences, University of Kentucky, Lexington, KY 40506, USA
}

Received January 22, 2008; Accepted February 19, 2008

\begin{abstract}
Wnt proteins control cell fate and differentiation during development. Alterations of the Wnt/ß-catenin signaling pathway and changes in wht gene expression are clearly associated with leukemia. The expression of human wnt13/ $w n t 2 b$ is complex as it involves alternative promoters and RNA splicing giving rise to Wnt13A, -B and -C mRNA isoforms, which encode proteins with different intracellular localizations and functions. We investigated the expression of the human wnt13 in relation to leukemic cell differentiation. Differentiated endothelial cells expressed the highest levels of Wnt13 mRNA isoforms among various endothelial and leukemic cell lines. The differentiation of U937 cells towards monocyte/macrophages resulted in an increase of Wnt13B and $-\mathrm{C}$ mRNAs while Wnt13A mRNAs were decreased. The differentiation of K562 cells towards megakaryocytes was accompanied with the up-regulation of all Wnt13 mRNA isoforms. In the two differentiation systems, Wnt13B and -C expression correlated with the expression of the MAF-B transcription factor. Our data demonstrate the differential regulation of wnt13 promoters and pinpoint a Wnt13 isoform switch during differentiation of the leukemic U937 cells towards the monocyte/macrophage lineage, thereby suggesting new players in this process.
\end{abstract}

\section{Introduction}

Wnt proteins are a family of highly conserved signaling factors controlling cell fate and differentiation during development (1). The spatio-temporal expression of wnt is tightly regulated during development with specific increases during patterning and organogenesis to becoming minimal if not undetectable

Correspondence to: Dr Catherine D. Mao, Graduate Center for Nutritional Sciences, University of Kentucky, Lexington, KY 40506, USA

E-mail: cdmao2@uky.edu

Present address: ${ }^{1}$ Department of Biomedical Sciences, Cornell University, Ithaca, NY 14853, USA

Key words: Wnt, differentiation, MAF-B, monocyte, erythrocyte, megakaryocyte, gene expression at later stages of embryogenesis, in neonates and adults (1). However, the re-expression of some wnt members in adult tissues has been associated with wound healing, tissue repair and regeneration $(2,3)$. In the latter, the $\mathrm{Wnt} / \mathrm{B}$-catenindependent signaling pathway was shown to control the selfrenewal properties of stem cells and the proliferation of early progenitor cells as well as their maintenance in an undifferentiated state (4-6).

Alterations of the $\mathrm{Wnt} / \mathrm{B}$-catenin signaling pathway are clearly associated with the tumorigenesis of tissues with a high renewal potential such as that of bone marrow-hematopoietic tissue (7). Although mutations of specific Wnt proteins have yet to be identified, changes in wnt gene expression have been demonstrated in various cancers including leukemia $(8,9)$. Moreover, different human wht genes show a complex organization and pattern of expression with alternative promoters and RNA splicing responsible for the expression of isoforms that differ only in their $\mathrm{N}$-terminal sequences and functions, such as human wnt13/wnt $2 b(10,11)$ and wnt16 (12). Notably, a differential up-regulation has been detected in human primary gastric cancer cells for Wnt13A/Wnt2B2 mRNA (13), and in basal cell carcinoma and pre-B acute lymphoblastoid leukemia for Wnt16B mRNA (8). Therefore, though less attention has thus far been paid to the regulation of wnt expression, such an analysis appears to be required to understand and define the respective role of individual Wnt proteins, if not individual Wnt isoforms, in the control of cell fate, tissue regeneration and tumorigenesis.

We have previously reported the expression of the $w n t 13 / w n t 2 b$ gene in human differentiated endothelial cells (EC), in which two alternative promoters gave rise to the transcription of Wnt13B and -C mRNAs and Wnt13A mRNA, respectively $(10,11)$, while an alternative skipping of exon 2 gave rise to Wnt13C mRNA (11). Notably, wnt13/wnt2b was also found to be expressed in human fetal hematopoietic cells and various leukemic cell lines, though in the earlier studies the different Wnt13 mRNA forms were not distinguished (14-16). We have previously observed that Wnt13B and -C mRNAs were the predominant forms in human differentiated EC (11), suggesting a possible switch of expression of the Wnt13 isoforms during endothelial and hematopoietic differentiation.

Leukemic cells represent early hematopoietic progenitors at different stages of commitment with self-renewal properties which fail to undergo terminal differentiation and apoptosis in situ (17). Various leukemic established cell lines 
recapitulate self-renewal and differentiation block in in vitro culture conditions while they can be differentiated in single or multiple cell lineages depending on the hematopoietic stage they arise from, such as the human monoblastic U937 leukemic cell line which is able to differentiate only towards the monocyte/macrophage lineage (18), and the multipotent K562 cell line which is able to differentiate towards either the erythroid lineage or megakaryocytes (19). During these early stages of hematopoietic cell differentiation, specific gene expression programs are driven by lineage-specific transcription factors $(20,21)$ and are thus characterized by specific correlation patterns of co-regulation among the lineagespecific transcription factors, signaling effectors and markers of differentiation that can be traced by hierarchical cluster analysis (22).

In the present study, we have employed real-time PCR analysis to determine the expression levels of Wnt13 mRNA isoforms and their correlation with the expression of hematopoietic differentiation markers.

\section{Materials and methods}

Cell culture. U937 (a gift from Dr G. Van Zant, University of Kentucky), THP1 (ATCC) and K562 cell lines (ATCC and a gift from Dr G. Van Zant) were cultured in RPMI-1640 supplemented with $10 \%$ heat-inactivated fetal bovine serum (FBS), $1 \%$ penicillin/streptomycin (Invitrogen, Carlsbad, CA) in a $5 \% \mathrm{CO}_{2}$ humidified atmosphere at $37^{\circ} \mathrm{C}$. Human umbilical vein endothelial cells (HUVEC) were isolated and grown on a fibronectin-coated plate as previously described (11). EAhy 926 (23) were cultured in DMEM (Invitrogen) supplemented with $5 \% \mathrm{FBS}$ and $1 \%$ penicillin/streptomycin.

Leukemic cell differentiation. Experimental cultures of U937 and K562 cells were initiated at densities between 0.5- and $4.5-\times 10^{5}$ cells $/ \mathrm{ml}$ and the cells were collected at various time points after treatment with or without differentiating agents. The differentiating agents, phorbol 12-myristate 13-acetate (PMA) (Calbiochem), all-trans retinoic acid (ATRA) and hemin (Sigma) were administered from stock solutions in dimethyl sulfoxide (DMSO) at a final concentration of $10 \mathrm{nM}$, 1 and $50 \mu \mathrm{M}$, respectively. The vehicle control was $10^{-2} \mu \mathrm{l} / \mathrm{ml}$ DMSO. Cell counts were monitored using a hemocytometer and viability was estimated using trypan blue exclusion. Phase contrast images of K562 and U937 cells after treatment with the differentiating agents were taken with an inverted microscope (Nikon, TE2000), with 20X objective to evaluate the morphological changes associated with differentiation.

RNA isolation, first strand DNA synthesis and real-time PCR. Total RNA was isolated using TRIzol reagent (Invitrogen). Total RNA $(2 \mu \mathrm{g})$ was DNase-I treated to remove any genomic DNA contaminations prior to the addition of $0.5 \mu \mathrm{g}$ of oligo(dT) $)_{12-18}$ primers and SuperScript III reverse transcriptase according to the manufacturer's protocol (Invitrogen). The real-time PCR was performed in duplicates using, per reaction, $34 \mathrm{ng}$ equivalents of total RNA, 10 pmoles of primers for highly expressed genes and 2.5 pmoles for low-expressing genes and the SYBR-Green kit (Applied Biosystems, Foster City, CA) in an ABI Prism ${ }^{\circledR} 7000$ sequence detection system (Applied-Biosystems). The primers used in this study are listed in Table I and the identity of the amplicons was verified by sequencing and restriction digests. To verify the accuracy and specificity of each real-time PCR reading, the dissociation curves were analyzed and the PCR products were resolved on $2 \%$ agarose gels.

Data and statistical analyses of the real-time PCR. For each target gene, the threshold cycle number $(\mathrm{Ct})$ was calculated with SDS v1.7 software (Applied Biosystems) and the duplicates averaged. The expression of $B$-actin mRNAs did not significantly vary in any of our experimental conditions and cell lines, and was used for normalization of the levels of the target mRNAs. The $\mathrm{Ct}$ differences $\left(\Delta \mathrm{Ct}=\mathrm{Ct}_{\text {target gene }}-\mathrm{Ct}_{B \text {-actin }}\right)$ of the target gene and $B$-actin mRNA levels were determined and the relative levels of target mRNA for each treatment were calculated using $\Delta \Delta \mathrm{Ct}\left(\Delta \Delta \mathrm{Ct}=\Delta \mathrm{Ct}_{\text {control }}-\Delta \mathrm{Ct}_{\text {treatment }}\right)$. Pair-wise plots were used to determine the normal distribution on a log scale of the various gene expression and statistical significance was determined using one-way ANOVA. To assess the correlation of the expression patterns, a hierarchical cluster analysis (22) was conducted by average linkage clustering and gene similarity metrics based on the Pearson correlation using SYSTAT 8.0 software.

\section{Results}

All three Wnt13 mRNA isoforms are expressed in human cells. The ability to accurately quantify the three Wnt13 transcripts is essential to following the specific regulation of each mRNA and hence of the corresponding Wnt13 protein forms $(10,11)$. Due to the limited differences in sequence and size between the three mRNA isoforms, real-time PCR is the best-suited method for their quantification. For this purpose, a common reverse primer located in exon 4 was designed, while the specific forward primers were chosen within the alternative exons 2 and 3 for Wnt13B and -A, respectively, and to encompass the exon 1-4 junction for Wnt13C (Fig. 1A). These specific Wnt13 mRNA isoform primer sets gave the expected amplicons by real-time PCR (Fig. 1B) allowing their use for the relative quantification of Wnt13 mRNA isoforms in various cell types ranging from primarydifferentiated HUVEC to undifferentiated multipotent leukemic K562 cells. Since the Ct values for $\beta$-actin mRNAs were similar from one cell line to another, the expression

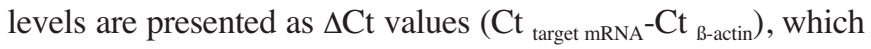
are inversely related to the RNA levels. Although the Wnt13 mRNA isoforms are present in the cell types investigated, their relative levels vary between them and cell types (Fig. 1C). The primary HUVEC display the highest levels of all Wnt13 mRNA isoforms in the order of Wnt13C $>$ Wnt13B $>$ Wnt13A, with Wnt13C being 16-fold more abundant than Wnt13A. In the opposite level range, the EAhy cell line, an established hybrid between HUVEC and A549 transformed lung epithelial cells (23), and the multipotent K562 cells have the lowest levels with 16- and 64-fold less Wnt13C and -B expression than HUVEC, respectively, though the order of Wnt13C> Wnt13B $>$ Wnt13A is conserved. In contrast, the monoblastic U937 cells present an unusual profile with Wnt13B mRNA levels being 100- and 400-fold lower than those of Wnt13A 
Table I. Primers used for real-time PCR quantification of the mRNA levels of Wntl3 isoforms and differentiation markers.

\begin{tabular}{lll}
\hline Gene & \multicolumn{1}{c}{ Forward primer sequence $\left(5^{\prime} \rightarrow 3^{\prime}\right)$} & Reverse primer sequence $\left(5^{\prime} \rightarrow 3^{\prime}\right)$ \\
\hline B actin & GGACTTCGAGCAAGAGATGG & AGCACTGTGTTGGCGTACAG \\
Wnt13A & CGTAGACACGTCCTGGTGGTA & GCATGATGTCTGGGTAACGC \\
Wnt13B & GATCCTTGAGGACGGCAGTA & GCATGATGTCTGGGTAACGC \\
Wntl3C & CTAAAAGTACATTGGGGCAC & GCATGATGTCTGGGTAACGC \\
MAF-B & CTGGCTTTCTGAACTTTGCGCGTT & AGGTCCGTCTGTCCTTCCTT \\
CDllb & GGGAAGTGGCAAGGAATGTA & CTGCGTGTGCTGTTCTTGT \\
PUl/Spi-1 & ATCAGAAGACCTGGTGCCCTATGA & TGAAGTTGTCTCGGCGAAGCTCT \\
gp91/Nox2 & TCACTTCCTCCACCAAAACC & GGGATTGGGCATTCCTTTAT \\
Caspase 4/5 & CACAGCCAGGGATATGGAGT & GCCTGGACAATGATGACCTT \\
IL8 8 CTCTGCACCCAGTTTCCTTG \\
GATA1 & GTGCAGTTTTGCCAAGGAGTG & TTCCAGATGCCTTGCGGTTT \\
GATA2 & AGAAGCGCCTGATTGTCAGT & CAGTTGACACACTCCCGGC \\
CD41/GPII & GTCACTGACGGAGAGCATGA & TCCACCTTGAGAGGGTTGAC \\
GPHA & AGGTGAGAGGGAGCAGAACA & GTCGGCGAATACCGTAAGAA \\
B/ globin & CAAACGGGACACATATGCAG & GCCACCACTTTCTGATAGGC \\
\hline
\end{tabular}

A

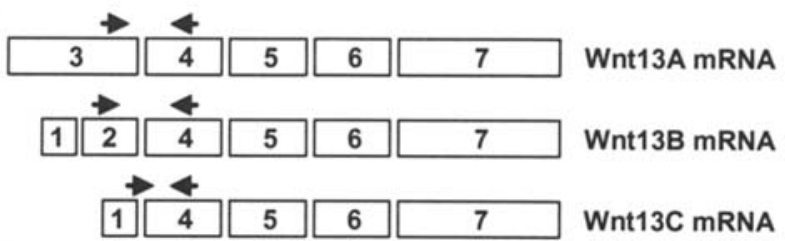

B

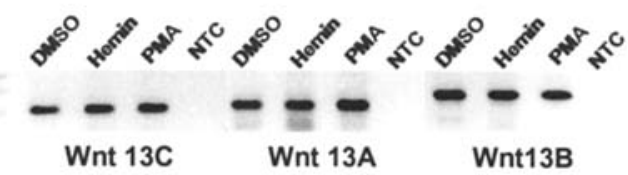

C

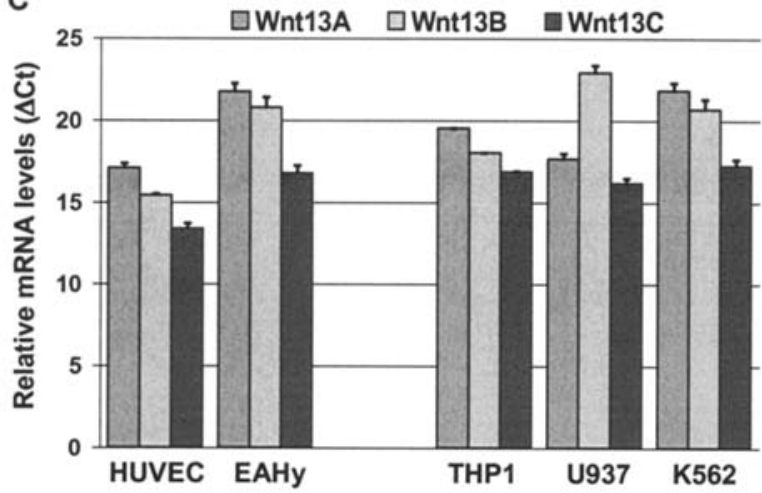

Figure 1. Expression of the Wnt13 mRNA isoforms in different human cell lines. (A) Schematic representation of human Wnt13 mRNAs and the position of the primers used (arrows) for assessing their expression. (B) Analysis of the real-time PCR products obtained from no template control (NTC) and from the cDNAs of K562 cells treated as indicated. (C) The relative levels of the Wnt13 mRNA isoforms were determined by real-time PCR using B-actin mRNA levels for normalization. The results are expressed as $\Delta \mathrm{Cts}$, which are inversely related to the mRNA amounts and represent the mean \pm SEM of at least four different experiments, except for THP1.

and -C mRNAs, respectively (Fig. 1C). The monocytic THP1 cells, which are more differentiated than U937 cells, present a more similar profile than does HUVEC though the levels of the three Wnt13 mRNA isoforms were still 10-fold lower (Fig. 1C).

The three Wnt13 mRNA isoforms are expressed in the human cell types we investigated, and although their levels tend to decrease in undifferentiated and transformed cell lines, the expression pattern Wnt13C $>$ Wnt13B $>W n t 13 A$ is conserved except in U937 cells. To further analyze the relationship between the expression of Wnt13 RNA isoforms and cell differentiation, we used the well-established differentiation systems of the monoblastic U937 cells towards monocyte/macrophages in the presence of PMA and ATRA (18), and of the erythroblastic K562 cells towards megakaryocytes with PMA (24) and erythrocytes with hemin (19). The changes in expression of Wnt13 mRNA isoforms during the differentiation of U937 and K562 cells towards these different hematopoietic lineages were assessed and then compared to those of lineage-specific transcription factors as well as differentiation and inflammation markers (Fig. 2). The expression of the transcription factor MAF-B was followed since its expression was associated with monocyte/macrophage $(25,26)$ and megakaryocytic differentiation $(27,28)$.

Switch of expression between Wnt13A and -B mRNA isoforms during the differentiation of U937 cell towards the monocytel macrophage lineage. Surprisingly, the expression of Wnt13B and -C mRNA isoforms increased significantly over time in U937 cells treated with DMSO only, of 100- and 500-fold for Wnt13B and 4- and 16-fold for Wnt13C at days 4 and 7, respectively, while the expression of Wnt13A remained unchanged or reduced (Fig. 2C). These increases of Wnt13B and $-3 \mathrm{C}$ mRNA levels were associated mainly with an increase of MAF-B and to a lesser extent to increases of the monocytic marker CD11b and of the inflammation marker caspases 4 and 5 at day 4 (Fig. 2C). As expected, PMA treatment induced a rapid cell cycle arrest in U937 cells as demonstrated by the absence of cell proliferation during a 4-day period (Fig. 2A) and characteristic morphological 

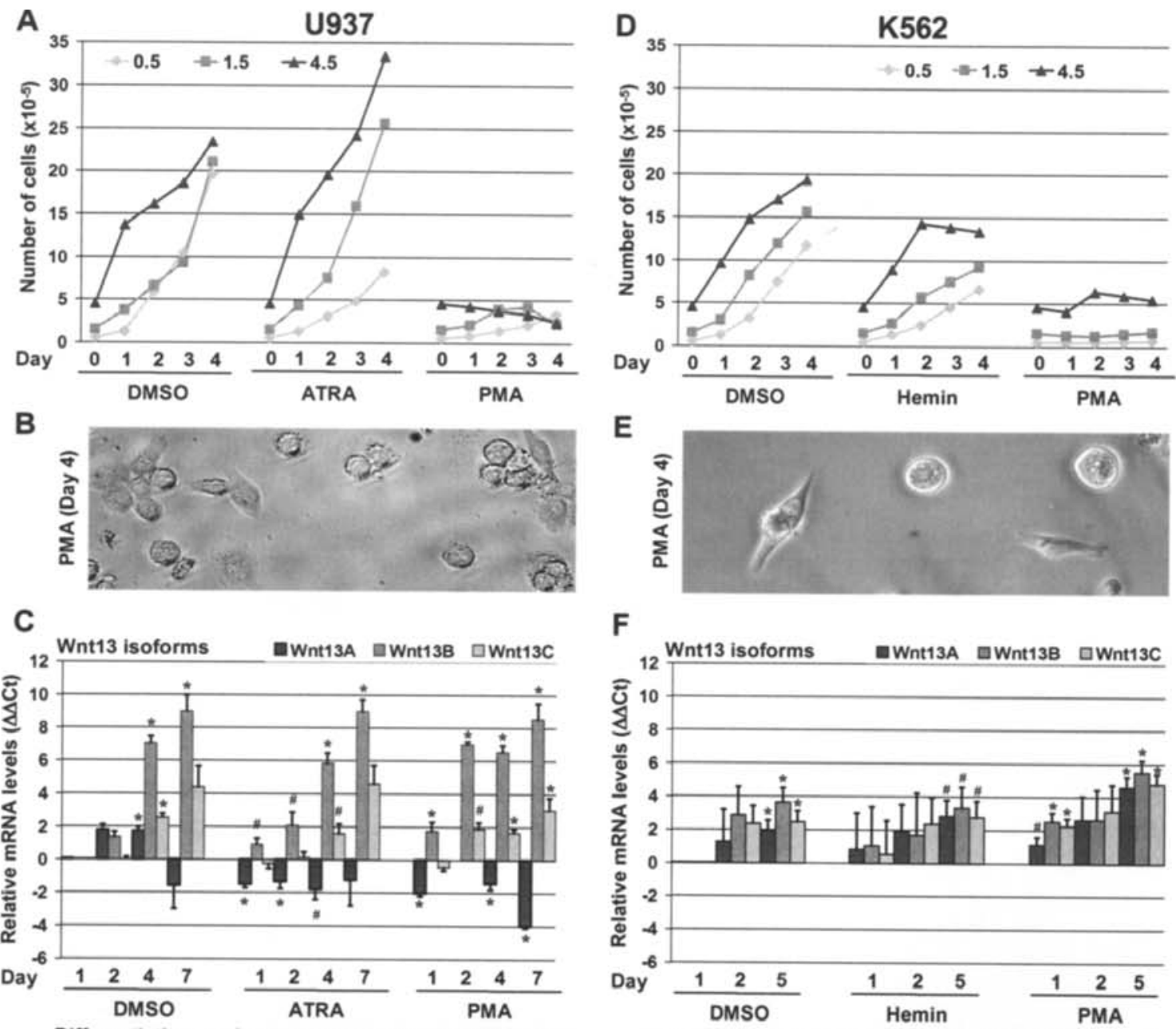

$\mathbf{F}$
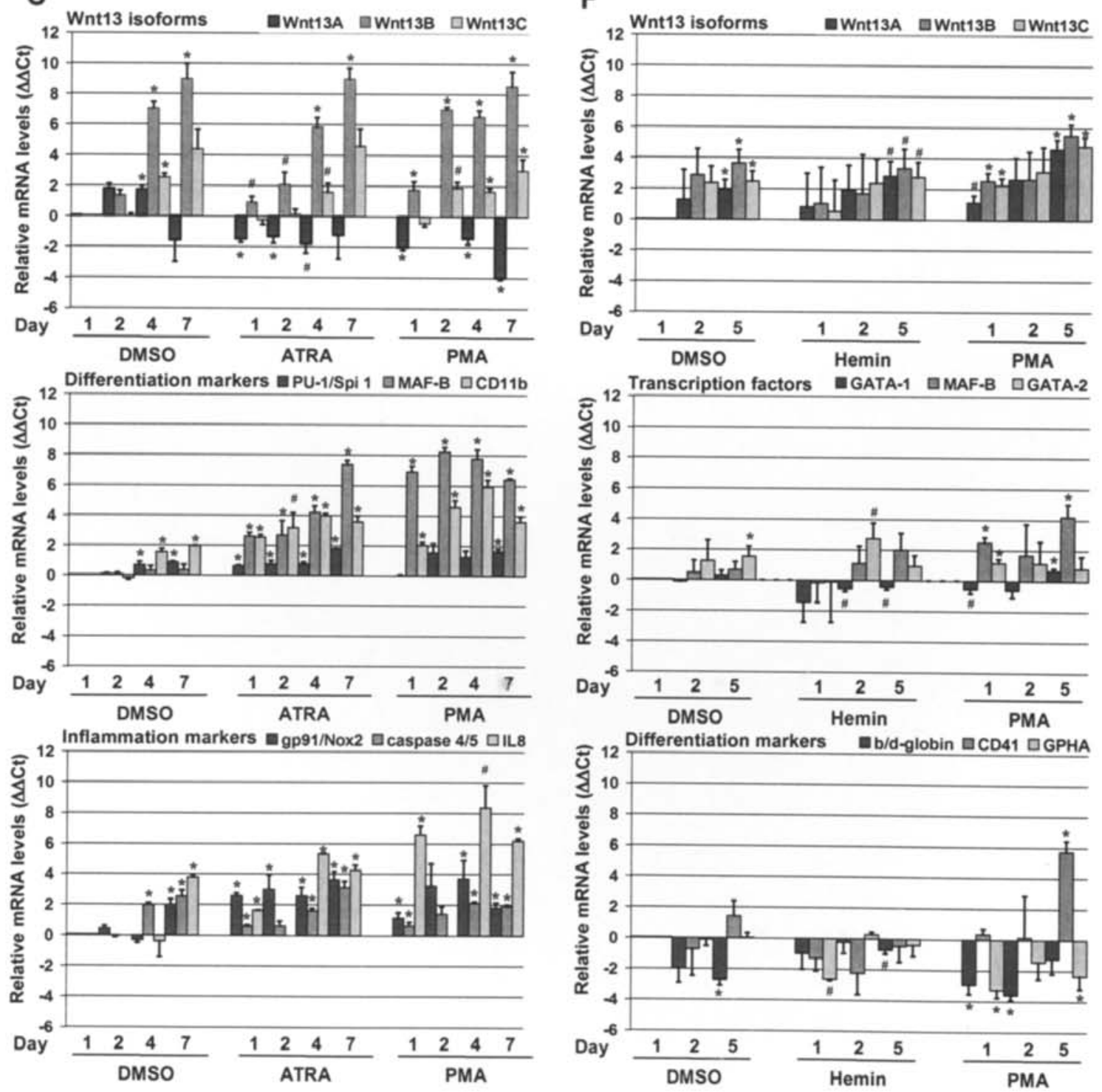

Figure 2. Differential regulation of Wnt13 isoforms during U937 and K562 cell differentiation. (A and D) The proliferative rate of U937 (A) and K562 (D) cells plated at $0.5-, 1.5$ - and $4.5-\mathrm{x} 10^{5}$ cells $/ \mathrm{ml}$ in the presence of DMSO $\left(10^{-2} \mu \mathrm{l} / \mathrm{ml}\right)$, PMA $(10 \mathrm{nM})$, ATRA $(1 \mu \mathrm{M})$ or hemin $(50 \mu \mathrm{M})$ was determined by cell counting at the indicated time after treatments. Representative experiments are shown. (B and E) Changes in the U937 (B) and K562 (E) morphology after four days of PMA treatment were assessed by phase contrast imaging at x20 magnification. Representative images are shown. (C and F) The expression of the differentiation markers and Wnt13 isoforms were assessed by real-time PCR in U937 (C) and K562 (F) cells plated at $1.5 \times 10^{5}$ cells $/ \mathrm{ml}$ and treated as above for the indicated time. The relative mRNA levels are expressed as $\Delta \Delta \mathrm{Ct}$ using the samples treated with DMSO for $24 \mathrm{~h}$ as controls. The mean $\pm \mathrm{SEM}$ obtained from at least three experiments is shown with ${ }^{*} \mathrm{p}<0.05$ and ${ }^{*} \mathrm{p}<0.01$ as compared to DMSO Day 1 (one-way ANOVA). 
changes of differentiated U937 cells, such as cell attachment to the substratum and spreading (Fig. 2B). These changes were accompanied by a rapid induction of the $\mathrm{CD} 11 \mathrm{~b}$ marker, with a 60-fold increase after 4 days of PMA treatment (Fig. 2C). MAF-B expression was increased by 100 -fold after only $24 \mathrm{~h}$ of PMA treatment. Following the kinetics of MAF-B upregulation, the expression of Wnt13B mRNA was highly increased, 100-fold, from 2 up to 7 days of treatment, while Wnt13C expression was moderately increased, 4- to 8-fold, and Wnt13A expression was decreased by $95 \%$ (Fig. 2C). Treatment with ATRA did not affect the proliferation of U937 cells (Fig. 2A), but was still inducing the expression of the CD11b marker (15-fold) with faster kinetics than the DMSO treatment (Fig. 2C). Concomitantly with the extent of U937 cell differentiation, ATRA treatment for two days was accompanied by increases of Wnt13B (8-fold) and MAF-B (8-fold) mRNA levels while the Wnt13A mRNA levels decreased by $75 \%$ (Fig. 2C). Thus, the unusual Wnt 13 expression pattern in U937 cells was reversed during their differentiation towards monocyte/macrophages by the combination of Wnt13A mRNA down-regulation and the substantial up-regulation of Wnt13B mRNA.

Up-regulation of the Wnt 13 mRNA isoforms during the differentiation of K562 cells towards megakaryocytes. A slight increase of the expression of the Wnt13 mRNA isoforms was observed over time in the K562 cells treated with DMSO, which was accompanied with a $75 \%$ decrease of the erythroid ß/ס-globin marker and a 4-fold increase of the megakaryocytic CD41 marker (Fig. 2F), indicating a spontaneous differentiation towards megakaryocytes. PMA treatment resulted in a rapid inhibition of K562 cell proliferation (Fig. 2D) and a 25-fold increase of the megakaryocytic marker CD41, while the expression of the erythroid markers, $\beta / \delta$ globin and glycophorin A, was decreased by $95 \%$ (Fig. 2F). In addition, morphological changes such as attachment and large intracellular vacuoles were observed (Fig. 2E). However, in contrast to the U937 cells where the MAF-B and Wnt13B mRNAs were highly increased after PMA treatment, the K562 cells presented only a slight increase of the Wnt13B and -C mRNAs (4-fold) and the MAF-B mRNA (8-fold) after one day of treatment (Fig. 2F). The PMA-induced megakaryocytic differentiation of K562 cells was accompanied with an increase of the Wnt13A mRNA levels (3-fold) (Fig. 2F). Hemin inhibited the proliferation of K562 cells after 2 days of treatment (Fig. 2A) and blunted the spontaneous differentiation of K562 cells towards megakaryocytes as indicated by the significant decrease of CD41 mRNA levels and to a smaller decrease of the $3 / \delta$-globin mRNAs (Fig. $2 \mathrm{~F}$ ). Despite these small features of differentiation, the 4- to 10fold increase of Wnt13 mRNA isoforms were similar in levels and kinetics to those obtained with DMSO treatment over time (Fig. 2F). These responses to hemin treatment were consistent in two different batches of K562 cells and reflected their lower frequency of chromosome Philadelphia and lesser sensitivity to erythrocyte-induced differentiation (29). Nonetheless, the absence of significant changes of Wnt13 expression in response to hemin reinforces the specificity of the changes observed in K562 cells, differentiated with PMA, and their association with MAF-B expression.
Wnt $13 B$ and $-C$ expression correlates with MAF-B expression. Our results in U937 and K562 cells pinpoint the association of Wnt13B and C with MAF-B expression rather than cell cycle arrest per se (Fig. 2A and D), cell apoptosis (not shown), or a particular differentiation state (Fig. 2C and F). To further establish the correlation between the expression of Wnt13B and $\mathrm{C}$ and MAF-B, we performed agglomerative hierarchical clustering (22) using the gene similarity metrics based on the Pearson correlation between the different $\Delta \mathrm{Ct}$ of the target mRNAs studied. Pattern similarities in gene expression in U937 and K562 cells have confirmed the co-regulation of Wnt13B and -C mRNA expression (Fig. 3A), and the control of Wnt13B and -C mRNA expression by a common promoter (11). Moreover, the transcription factor MAF-B clustered with Wnt13B and -C in U937 in the control and differentiated cells, while such clustering was only seen in the K562 cells differentiated with PMA (Fig. 3A). This correlation between MAF-B and Wnt13B mRNA expression in U937 cells was observed as early as $3 \mathrm{~h}$ after PMA treatment with an 8- and 3-fold increase, respectively, while the levels of Wnt13C mRNA remained unchanged (Fig. 3B). Based on the initial difference of Wnt13B and -C mRNAs in U937 cells (400-fold, Fig 1C), the latter is in agreement with a transcriptional activation of the Wnt13B and -C promoter and/or an alternative RNA splicing switch favoring Wnt13B mRNA. On the other hand, Wnt13A expression was clustered with neither Wnt13B and $-\mathrm{C}$ nor any of the transcription factors tested in the U937 and K562 cells with the exception of K562 cells treated with hemin (Fig. 3A). The absence of correlation between Wnt13A, and Wnt13B and -C mRNA expression confirms the existence of a second promoter in the wnt13 gene and its differential regulation.

\section{Discussion}

Taken together, our results confirm the presence of two promoters in the human Wnt13 gene and demonstrate their differential regulation, particularly during the differentiation of the monoblastic U937 leukemia cell line towards monocyte/ macrophage. Moreover, unlike Wnt13A expression, the expression of Wnt13B and -C mRNAs was highly correlated with the expression of the transcription factor MAF-B in various cell lines and differentiation systems. Although Wnt13A/Wnt2b was shown to be downstream of the MAF-B cascade during the inner ear patterning and eye development in the zebrafish, newt and mouse $(2,30,31)$, this is the first report specifically linking the expression of human Wnt13B and $-\mathrm{C}$ isoforms with the MAF-B transcription factor during leukemic cell differentiation, which emphasizes the conservation of wnt $13 / w n t 2 b$ and maf-B association in different developmental and differentiation systems.

We showed herein that all three Wnt13 mRNA isoforms are present in all the human cell types we investigated, and although their levels tend to decrease in undifferentiated and transformed cell lines, the expression pattern Wnt13C> Wnt13B $>$ Wnt13A is conserved in most of them (Fig. 1C). Only the monoblastic U937 leukemia cells presented an unusual pattern with Wnt13C $>$ Wnt13A $>$ Wnt13B (Fig. 1C), which was reversed during their differentiation towards monocyte/macrophages by the combination of Wnt13A 
A

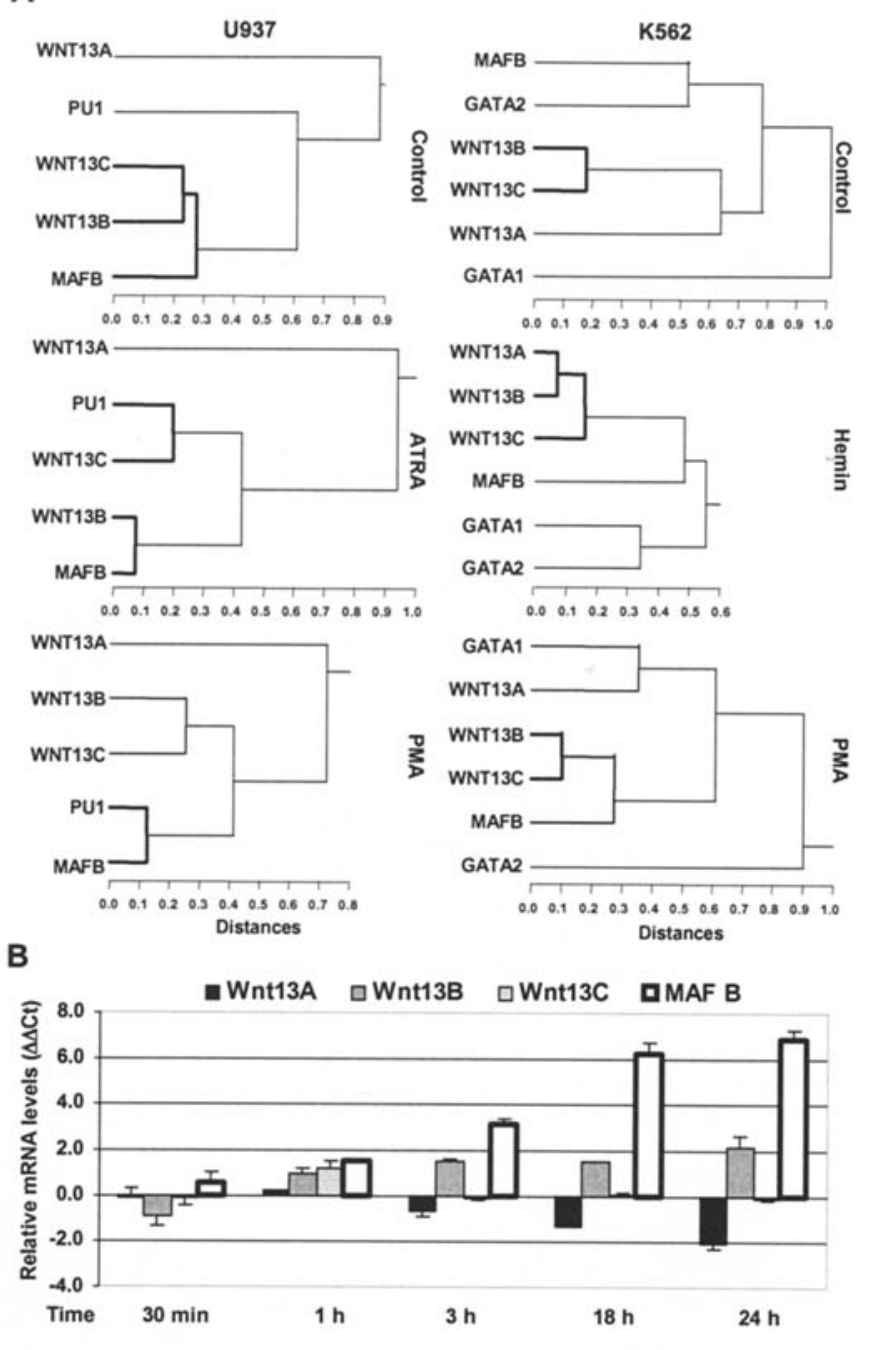

Figure 3. Wnt13B and -C mRNA expression correlates with MAF-B mRNA expression. (A) The treatment-dependent expression profiles of Wnt13 isoforms and several transcription factors in U937 and K562 cells were hierarchically clustered based on the Pearson correlation from 130 samples/ cell line. (B) The acute effects of PMA $(10 \mathrm{nM})$ on the expression of MAF$\mathrm{B}$ and Wnt13 isoform mRNAs in U937 cells were determined by real-time PCR. The results are shown as $\Delta \Delta C t$ using DMSO-treated cells at the same time point as the control (mean \pm SEM of two independent experiments).

mRNA down-regulation and the substantial up-regulation of Wnt13B mRNA (Fig. 2A). As expected with a common promoter controlling Wnt13B and -C mRNA expression (11), there was a high correlation of Wnt13C and -B mRNA expression even in the U937 cells (Fig. 3A). Similarly, the absence of correlation between Wnt13A with Wnt13B and -C mRNA expression (Fig. 3A) strengthens the existence of the second promoter Wnt13 gene and its differential regulation. Besides transcriptional regulation, additional mechanisms probably occur to explain the difference in Wnt13C and -B mRNA levels in U937 cells (400-fold, Fig. 1C) and its disappearance after differentiation (8-fold) such as regulated RNA-splicing events (32).

Another human wnt gene, wnt 16, presents a similar genomic organization with two promoters responsible for the transcription of alternative exons containing divergent 5'untranslated region and coding for different N-termini (12). Similarly, the expression of the resulting Wnt16A and -B mRNA isoforms are differentially regulated, with Wnt16B mRNA expression being associated with pre-B acute lymphoblastoid leukemia $(8,33)$. Increased levels of Wnt13A mRNA have been noted in human primary gastric cancer cell lines (13). Among the leukemia cell lines tested, only the unusual pattern of expression of the Wnt13A and -B isoforms in U937 cells with high levels of Wnt13A mRNAs versus Wnt13B mRNA levels (Fig. 1C) and their switch of expression during the ATRA- and PMA-induced differentiation (Fig. 2A), may suggest a possible involvement of Wnt13 in the U937transformed phenotype. The expression of Wnt13A/Wnt2B2 in hematopoietic stem cells was associated with an increase of proliferation of erythroid and myeloid colonies and a decrease of their differentiation (14). Similarly, the expression of Wnt13A/Wnt2b during development of the eye in the mouse and chicken was associated with activation of the $\beta$ catenin signaling pathway, proliferation and inhibition of retinal progenitor cell differentiation (34-36). On the other hand, a decrease of Wnt13B and -C mRNA expression may participate in the resistance of leukemic cells to apoptosis $(37,38)$. The expression of the nuclear $\mathrm{Wnt} 13 \mathrm{~B}$ and $-\mathrm{C}$ forms have recently been shown to increase the sensitivity of differentiated endothelial cells to $\mathrm{TNF} \alpha$-induced apoptosis (11), suggesting that the up-regulation of Wnt13B and -C mRNA during differentiation may be involved in the increased susceptibility to apoptosis of terminally differentiated cells $(37,38)$.

However, the increased expression of Wnt13B and -C mRNAs during the differentiation of U937 and K562 cells was neither correlated with apoptosis (not shown) nor cell cycle arrest per se (Fig. 2), but with the expression of the transcription factor MAF-B as demonstrated by similar kinetics and the amplitudes of variations (Figs. 2-3B) as well as by unsupervised hierarchical clustering (Fig. 3A). Notably, the de-regulation of $m a f B$ expression is associated with $2 \%$ of the multiple myeloma via translocation of the immunoglobulin locus $(39,40)$. MAF-B is a bZIP transcription factor belonging to the large MAF sub-class, which is able to act either as a repressor or an activator depending on its interacting partners in various differentiation processes. MAF-B interaction with ets transcription factor appears to be required for the down-regulation of erythroid genes such as B-globin (28) and the up-regulation of the CD41 marker during the megakaryocytic differentiation (27). Moreover, MAF-B is crucial for the differentiation of non-adherent macrophages and expression of the macrophage marker F4/80 (41) as well as for the control of the balance between dendritic and monocytic differentiation $(25,26,42)$. The fact that the factors involved in a specific cellular process display similar gene expression patterns reinforces the possibility that the Wnt13 isoforms constitute additional players in the differentiation process towards monocyte/macrophages.

\section{Acknowledgements}

We are thankful to Dr Gary Van Zant for the kind gift of the hematopoietic U937 and K562 cell lines. This work was supported by NIH grant HL68698 to C.D.M. 


\section{References}

1. Moon RT, Brown JD and Torres M: WNTs modulate cell fate and behavior during vertebrate development. Trends Genet 13: 157-162, 1997.

2. Hayashi T, Mizuno N, Takada R, Takada S and Kondoh H: Determinative role of Wnt signals in dorsal iris-derived lens regeneration in newt eye. Mech Dev 123: 793-800, 2006.

3. Fathke C, Wilson L, Shah K, Kim B, Hocking A, Moon R and Isik F: Wnt signaling induces epithelial differentiation during cutaneous wound healing. BMC Cell Biol 7: 4, 2006.

4. Reya T, Duncan AW, Ailles L, Domen J, Scherer DC, Willert K, Hintz L, Nusse R and Weissman IL: A role for Wnt signalling in self-renewal of haematopoietic stem cells. Nature 423: 409-414, 2003.

5. Kawakami Y, Rodriguez Esteban C, Raya M, Kawakami H, Marti M, Dubova I and Izpisua Belmonte JC: Wnt/beta-catenin signaling regulates vertebrate limb regeneration. Genes Dev 20: 3232-3237, 2006

6. Polesskaya A, Seale P and Rudnicki MA: Wnt signaling induces the myogenic specification of resident CD45+ adult stem cells during muscle regeneration. Cell 113: 841-852, 2003.

7. Staal FJ and Clevers HC: WNT signalling and haematopoiesis: a WNT-WNT situation. Nat Rev Immunol 5: 21-30, 2005.

8. McWhirter JR, Neuteboom ST, Wancewicz EV, Monia BP, Downing JR and Murre C: Oncogenic homeodomain transcription factor E2A-Pbx1 activates a novel WNT gene in pre-B acute lymphoblastoid leukemia. Proc Natl Acad Sci USA 96: 11464-11469, 1999.

9. Roman-Gomez J, Cordeu L, Agirre X, Jimenez-Velasco A, San Jose-Eneriz E, Garate L, Calasanz MJ, Heiniger A, Torres A and Prosper F: Epigenetic regulation of Wnt-signaling pathway in acute lymphoblastic leukemia. Blood 109: 34623469, 2007 .

10. Katoh M, Kirikoshi H, Saitoh T, Sagara N and Koike J: Alternative splicing of the WNT-2B/WNT-13 gene. Biochem Biophys Res Commun 275: 209-216, 2000.

11. Struewing IT, Toborek A and Mao CD: Mitochondrial and nuclear forms of Wnt13 are generated via alternative promoters, alternative RNA splicing, and alternative translation start sites. J Biol Chem 281: 7282-7293, 2006.

12. Fear MW, Kelsell DP, Spurr NK and Barnes MR: Wnt-16a, a novel Wnt-16 isoform, which shows differential expression in adult human tissues. Biochem Biophys Res Commun 278: 814-820, 2000 .

13. Katoh M, Kirikoshi H, Terasaki H and Shiokawa K: WNT2B2 mRNA, up-regulated in primary gastric cancer, is a positive regulator of the WNT-beta-catenin-TCF signaling pathway. Biochem Biophys Res Commun 289: 1093-1098, 2001.

14. Van Den Berg DJ, Sharma AK, Bruno E and Hoffman R: Role of members of the Wnt gene family in human hematopoiesis. Blood 92: 3189-3202, 1998 .

15. Lako M, Lindsay S, Lincoln J, Cairns PM, Armstrong L and Hole N: Characterisation of Wnt gene expression during the differentiation of murine embryonic stem cells in vitro: role of Wnt3 in enhancing haematopoietic differentiation. Mech Dev 103: 49-59, 2001

16. Dosen G, Tenstad E, Nygren MK, Stubberud H, Funderud S and Rian E: Wnt expression and canonical Wnt signaling in human bone marrow B lymphopoiesis. BMC Immunol 7: 13, 2006.

17. Tenen DG: Disruption of differentiation in human cancer: AML shows the way. Nat Rev Cancer 3: 89-101, 2003.

18. Sundstrom $\mathrm{C}$ and Nilsson K: Establishment and characterization of a human histiocytic lymphoma cell line (U-937). Int J Cancer 17: 565-577, 1976.

19. Tsiftsoglou AS, Pappas IS and Vizirianakis IS: Mechanisms involved in the induced differentiation of leukemia cells. Pharmacol Ther 100: 257-290, 2003.

20. Orkin SH: Diversification of haematopoietic stem cells to specific lineages. Nat Rev Genet 1: 57-64, 2000.

21. Rosenbauer F and Tenen DG: Transcription factors in myeloid development: balancing differentiation with transformation. Nat Rev Immunol 7: 105-117, 2007.

22. Eisen MB, Spellman PT, Brown PO and Botstein D: Cluster analysis and display of genome-wide expression patterns. Proc Natl Acad Sci USA 95: 14863-14868, 1998.
23. Edgell CJ, McDonald CC and Graham JB: Permanent cell line expressing human factor VIII-related antigen established by hybridization. Proc Natl Acad Sci USA 80: 3734-3737, 1983.

24. Tetteroo PA, Massaro F, Mulder A, Schreuder-van Gelder R and von dem Borne AE: Megakaryoblastic differentiation of proerythroblastic K562 cell-line cells. Leuk Res 8: 197-206, 1984.

25. Kelly LM, Englmeier U, Lafon I, Sieweke MH and Graf T: $\mathrm{MafB}$ is an inducer of monocytic differentiation. EMBO J 19: 1987-1997, 2000.

26. Gemelli C, Montanari M, Tenedini E, Zanocco Marani T, Vignudelli T, Siena M, Zini R, Salati S, Tagliafico E, Manfredini R, Grande A and Ferrari S: Virally mediated MafB transduction induces the monocyte commitment of human CD34+ hematopoietic stem/progenitor cells. Cell Death Differ 13: 1686-1696, 2006.

27. Sevinsky JR, Whalen AM and Ahn NG: Extracellular signalregulated kinase induces the megakaryocyte GPIIb/CD41 gene through MafB/Kreisler. Mol Cell Biol 24: 4534-4545, 2004.

28. Sieweke MH, Tekotte H, Frampton J and Graf T: MafB is an interaction partner and repressor of Ets-1 that inhibits erythroid differentiation. Cell 85: 49-60, 1996.

29. Dimery IW, Ross DD, Testa JR, Gupta SK, Felsted RL, Pollak A and Bachur NR: Variation amongst K562 cell cultures. Exp Hematol 11: 601-610, 1983.

30. Choo D, Ward J, Reece A, Dou H, Lin Z and Greinwald J: Molecular mechanisms underlying inner ear patterning defects in kreisler mutants. Dev Biol 289: 308-317, 2006.

31. Lin Z, Cantos R, Patente M and Wu DK: Gbx2 is required for the morphogenesis of the mouse inner ear: a downstream candidate of hindbrain signaling. Development 132: 2309-2318, 2005.

32. Pascual M, Vicente M, Monferrer L and Artero R: The Muscleblind family of proteins: an emerging class of regulators of developmentally programmed alternative splicing. Differentiation 74: 65-80, 2006.

33. Teh MT, Blaydon D, Ghali LR, Briggs V, Edmunds S, Pantazi E, Barnes MR, Leigh IM, Kelsell DP and Philpott MP: Role for WNT16B in human epidermal keratinocyte proliferation and differentiation. J Cell Sci 120: 330-339, 2007.

34. Kubo F, Takeichi $M$ and Nakagawa S: Wnt2b inhibits differentiation of retinal progenitor cells in the absence of Notch activity by down-regulating the expression of proneural genes. Development 132: 2759-2770, 2005.

35. Cho SH and Cepko CL: Wnt2b/beta-catenin-mediated canonical Wnt signaling determines the peripheral fates of the chick eye. Development 133: 3167-3177, 2006.

36. Jasoni C, Hendrickson A and Roelink H: Analysis of chicken Wnt-13 expression demonstrates coincidence with cell division in the developing eye and is consistent with a role in induction. Dev Dyn 215: 215-224, 1999.

37. Launay S, Hermine O, Fontenay M, Kroemer G, Solary E and Garrido C: Vital functions for lethal caspases. Oncogene 24: 5137-5148, 2005.

38. Domen J: The role of apoptosis in regulating hematopoietic stem cell numbers. Apoptosis 6: 239-252, 2001.

39. Hanamura I, Lida S, Akano Y, Hayami Y, Kato M, Miura K, Harada S, Banno S, Wakita A, Kiyoi H, Naoe T, Shimizu S, Sonta SI, Nitta M, Taniwaki M and Ueda R: Ectopic expression of MAFB gene in human myeloma cells carrying $(14 ; 20)$ (q32;q11) chromosomal translocations. Jpn J Cancer Res 92: 638-644, 2001.

40. Boersma-Vreugdenhil GR, Kuipers J, Van Stralen E, Peeters T, Michaux L, Hagemeijer A, Pearson PL, Clevers HC and Bast BJ: The recurrent translocation $\mathrm{t}(14 ; 20)(\mathrm{q} 32 ; \mathrm{q} 12)$ in multiple myeloma results in aberrant expression of MAFB: a molecular and genetic analysis of the chromosomal breakpoint. $\mathrm{Br} \mathrm{J}$ Haematol 126: 355-363, 2004.

41. Moriguchi T, Hamada M, Morito N, Terunuma T, Hasegawa K, Zhang C, Yokomizo T, Esaki R, Kuroda E, Yoh K, Kudo T, Nagata M, Greaves DR, Engel JD, Yamamoto $M$ and Takahashi S: MafB is essential for renal development and F4/80 expression in macrophages. Mol Cell Biol 26: 5715-5727, 2006.

42. Bakri Y, Sarrazin S, Mayer UP, Tillmanns S, Nerlov C, Boned A and Sieweke MH: Balance of MafB and PU.1 specifies alternative macrophage or dendritic cell fate. Blood 105: 2707-2716, 2005. 